ANNALES

POLONICI MATHEMATICI

$90.3(2007)$

\title{
Complex surfaces in $\mathbb{C}^{4}$ with recurrent shape operators
}

\author{
by Pawee Witowicz (Rzeszów)
}

\begin{abstract}
We study complex affine surfaces in $\mathbb{C}^{4}$ with the transversal bundle defined by Nomizu and Vrancken. We classify the surfaces that have recurrent shape operators and parallel transversal metric.
\end{abstract}

1. Preliminaries. The purpose of this paper is to characterize a class of complex surfaces holomorphically immersed in four-dimensional affine space $\mathbb{C}^{4}$. An equiaffine structure for real surfaces in $\mathbb{R}^{4}$ was found by Nomizu and Vrancken ([NV]) and further investigations showed that the construction leads to natural geometric properties. In [W] a unique equiaffine transversal bundle is also obtained in the complex case. It has the same properties as the bundle constructed in $[\mathrm{NV}]$ and is holomorphic. There are three local pseudometrics in this bundle and we denote each of them by $g^{\perp}$. They differ only by a constant factor. A given $g^{\perp}$-null transversal frame $\left\{\xi_{1}, \xi_{2}\right\}$ determines shape operators $S_{1}$ and $S_{2}$ which are local endomorphisms of the tangent bundle. Let $\nabla$ and $\nabla^{\perp}$ be the connections in the tangent and normal bundles, respectively.

We recall that the shape operators are recurrent if there exist 1-forms $\alpha_{1}$ and $\alpha_{2}$ such that $\nabla S_{j}=\alpha_{j} \otimes S_{j}$. We, however, consider a special case $\nabla S_{j}=(-1)^{j-1} d f \otimes S_{j}$ because this condition does not depend on the frame $\left\{\xi_{1}, \xi_{2}\right\}$. We prove a classification theorem under the additional condition $\nabla^{\perp} g^{\perp}=0$.

We now state the main theorem of the paper.

TheOREM 1.1. Let $M$ be a non-degenerate complex surface in $\mathbb{C}^{4}$ satisfying the conditions:

1) in a neighbourhood of an arbitrary point $p$ of the surface, for every holomorphic null normal frame $\left\{\xi_{1}, \xi_{2}\right\}$ there is a local function $f$ such

2000 Mathematics Subject Classification: Primary 53A15.

Key words and phrases: affine immersion, surface, shape operator.

Supported by the KBN grant 1P03A03426. 
that

$$
\nabla S_{1}=d f \otimes S_{1}, \quad \nabla S_{2}=-d f \otimes S_{2},
$$

2) $\nabla^{\perp} g^{\perp}=0$.

Then there exists an open and dense set $U$ in $M$ which is locally affinely equivalent to one of the following surfaces:

(a) $x(u, v)=\left(u, v, u^{2}, v^{2}\right)$,

(b) $x(u, v)=u \cdot\left(1, v, v^{2}, u^{2}\right)$,

(c) $x(u, v)=\left(u^{3}+3 u^{2} v, v^{3}+3 u v^{2}, u-v, u v\right)$,

(d) $x(u, v)=(\cosh u)^{2 / 3} \cdot\left(1, v, v^{2}, \int(\cosh s)^{-5 / 3} d s\right)$,

(e) $x(u, v)=u^{-2} \cdot\left(1, u^{5}, v, \frac{1}{2} v^{2}\right)$.

(f) $x(u, v)=(\sinh (u), \cosh (u), \sinh (v), \cosh (v))$,

(g) $x(u, v)=\left(v, \frac{1}{2} v^{2}, f_{1}(u), f_{1}(u) \int^{u} \frac{d s}{\left(f_{1}^{\prime}(s)\right)^{2}}-\int^{u} \frac{f_{1}(s) d s}{\left(f_{1}^{\prime}(s)\right)^{2}}\right)$,

where $f_{1}$ is an arbitrary holomorphic function defined in a neighbourhood of zero and such that $f_{1}^{\prime}(0) \neq 0$ and $f^{(3)}(0) \neq 0$. Conversely, all these surfaces satisfy conditions 1 ) and 2).

Let $M$ be a two-dimensional complex submanifold of $\mathbb{C}^{4}$. This means that there exists an immersion $f: M \rightarrow \mathbb{C}^{4}$ which is holomorphic in the sense that $f_{*} J X=J f_{*} X$ for the complex structure $J$. Each tangent space $T_{x} M$ has a natural structure of a complex vector space with the multiplication by $i$ given by $J$. Let the vector fields, connections, bilinear and linear forms and functions used in the paper be of class $\mathcal{C}_{\mathbb{R}}^{\infty}$ unless otherwise stated (see [OI], [OII], compare also another approach in [A], [DVV]). Since our considerations are local and we can identify the complex structures on $M$ and on $\mathbb{C}^{4}$, we can also identify $M$, as a complex manifold, with its image in $\mathbb{C}^{4}$.

Let $\sigma$ denote a transversal complex plane bundle, that is, $\mathbb{C}^{4}=\sigma_{x} \oplus T_{x} M$ over $\mathbb{C}$. Let $\xi_{1}, \xi_{2}$ be transversal vector fields that span $\sigma$ locally over $\mathbb{C}$. Capital letters $X, Y, Z$ etc. will denote tangent vector fields. If $D$ denotes the standard affine connection on $\mathbb{C}^{4}$, then we have

$$
\begin{aligned}
& D_{X} Y=\nabla_{X} Y+h^{1}(X, Y) \xi_{1}+h^{2}(X, Y) \xi_{2}, \\
& D_{X} \xi_{j}=-S_{j}(X)+\tau_{j}^{1}(X) \xi_{1}+\tau_{j}^{2}(X) \xi_{2},
\end{aligned}
$$

for $j=1,2$, where the connection $\nabla$ is determined by the condition $\nabla_{X} Y \in T M$ and the shape operators $S_{j}$ by the condition $S_{j}(X) \in T M$. Then $\nabla$ is a torsion-free linear connection compatible with $J$ (and therefore called a complex connection). We can also see that both $h^{j}$ are $\mathbb{C}$ valued and $\mathbb{C}$-bilinear 2 -forms, called the second fundamental forms, $S_{j}$ 
are $(1,1)-\mathbb{R}$-linear tensors and $\tau_{k}^{j}$ are $\mathbb{C}$-valued, $\mathbb{R}$-linear 1 -forms (see $[\mathrm{KN}]$, $[\mathrm{OI}],[\mathrm{OII}])$. We also define a complex-valued $\mathbb{C}$-linear skew-symmetric 2-form $\theta$ by $\theta(X, Y)=\operatorname{Det}\left[X, Y, \xi_{1}, \xi_{2}\right]$, where Det denotes the usual determinant in $\mathbb{C}^{4}$. Just as in real geometry $([\mathrm{NV}])$ we have the following equations of Gauss (1.3), Codazzi ((1.4)-(1.7)) and Ricci ((1.8)-(1.11)):

$$
\begin{gathered}
R(X, Y) Z=h^{1}(Y, Z) S_{1} X+h^{2}(Y, Z) S_{2} X \\
-h^{1}(X, Z) S_{1} Y-h^{2}(X, Z) S_{2} Y, \\
\left(\nabla_{X} h^{1}\right)(Y, Z)+\tau_{1}^{1}(X) h^{1}(Y, Z)+\tau_{2}^{1}(X) h^{2}(Y, Z)
\end{gathered}
$$

is symmetric in $X, Y$ and $Z$,

$$
\left(\nabla_{X} h^{2}\right)(Y, Z)+\tau_{1}^{2}(X) h^{1}(Y, Z)+\tau_{2}^{2}(X) h^{2}(Y, Z)
$$

is symmetric in $X, Y$ and $Z$,

$$
\begin{gathered}
\left(\nabla_{X} S_{1}\right) Y-\left(\nabla_{Y} S_{1}\right) X \\
=-\tau_{1}^{1}(Y) S_{1} X+\tau_{1}^{1}(X) S_{1} Y-\tau_{1}^{2}(Y) S_{2} X+\tau_{1}^{2}(X) S_{2} Y, \\
\left(\nabla_{X} S_{2}\right) Y-\left(\nabla_{Y} S_{2}\right) X \\
=-\tau_{2}^{1}(Y) S_{1} X+\tau_{2}^{1}(X) S_{1} Y-\tau_{2}^{2}(Y) S_{2} X+\tau_{2}^{2}(X) S_{2} Y, \\
h^{1}\left(X, S_{1} Y\right)-h^{1}\left(Y, S_{1} X\right) \\
=d \tau_{1}^{1}(X, Y)+\tau_{1}^{2}(Y) \tau_{2}^{1}(X)-\tau_{2}^{1}(Y) \tau_{1}^{2}(X), \\
h^{2}\left(X, S_{1} Y\right)-h^{2}\left(Y, S_{1} X\right) \\
=d \tau_{1}^{2}(X, Y)+\tau_{1}^{1}(Y) \tau_{1}^{2}(X)-\tau_{1}^{2}(Y) \tau_{1}^{1}(X) \\
\quad+\tau_{1}^{2}(Y) \tau_{2}^{2}(X)-\tau_{2}^{2}(Y) \tau_{1}^{2}(X), \\
h^{2}\left(X, S_{2} Y\right)-h^{2}\left(Y, S_{2} X\right) \\
=d \tau_{2}^{2}(X, Y)+\tau_{2}^{1}(Y) \tau_{1}^{2}(X)-\tau_{1}^{2}(Y) \tau_{2}^{1}(X), \\
h^{1}\left(X, S_{2} Y\right)-h^{1}\left(Y, S_{2} X\right) \\
=d \tau_{2}^{1}(X, Y)+\tau_{1}^{1}(X) \tau_{2}^{1}(Y)-\tau_{2}^{1}(X) \tau_{1}^{1}(Y) \\
\quad+\tau_{2}^{1}(X) \tau_{2}^{2}(Y)-\tau_{2}^{2}(X) \tau_{2}^{1}(Y)
\end{gathered}
$$

Let $u=\left\{X_{1}, X_{2}\right\}$ be a local frame of class $\mathcal{C}_{\mathbb{R}}^{\infty}$ on a neighbourhood $U$ of a point $p \in M$. Define a symmetric bilinear form $G_{u}$ by

(1.12) $2 G_{u}(Y, Z)$

$$
=\operatorname{Det}\left[X_{1}, X_{2}, D_{Y} X_{1}, D_{Z} X_{2}\right]+\operatorname{Det}\left[X_{1}, X_{2}, D_{Z} X_{1}, D_{Y} X_{2}\right]
$$

We call a surface non-degenerate if the form $G_{u}$ is non-degenerate (which does not depend on the choice of $u$; see [W]). From now on we will assume that the surface is non-degenerate. In a sufficiently small neighbourhood of each point we can define three branches of a $\mathbb{C}$-valued, $\mathbb{C}$-bilinear symmetric form

$$
g_{u}(Y, Z)=G_{u}(Y, Z)\left(\operatorname{det}_{u} G_{u}\right)^{-1 / 3}
$$


The set of three branches of $g_{u}$ is independent of the choice of $u$. In this way we get locally three complex-valued metrics which we denote by $g$. We call each of them an affine metric on $M$. From now on we fix an affine metric in a sufficiently small neighbourhood of a given point. We will work with null frames with respect to the affine metric $g$, that is, the frames $\left\{X_{1}, X_{2}\right\}$ satisfying $g\left(X_{1}, X_{2}\right)=1$ and $g\left(X_{j}, X_{j}\right)=0$ for $j=1,2$. The following theorem associates a unique transversal frame to each null tangent frame (see $[\mathrm{NV}],[\mathrm{W}])$.

TheOREM 1.2. Let $\sigma$ be a transversal plane bundle and $\left\{X_{1}, X_{2}\right\}$ be a null tangent frame. Then there exists a unique local transversal frame $\left\{\xi_{1}, \xi_{2}\right\}$ in $\sigma$ such that

$$
\operatorname{Det}\left[X_{1}, X_{2}, \xi_{1}, \xi_{2}\right]=-2 \text {, }
$$

and the second fundamental forms have the following matrices in the basis $\left\{X_{1}, X_{2}\right\}$ :

$$
h^{1}=\left[\begin{array}{ll}
1 & 0 \\
0 & 0
\end{array}\right], \quad h^{2}=\left[\begin{array}{ll}
0 & 0 \\
0 & 1
\end{array}\right] .
$$

From now on we will call the frame $\left\{\xi_{1}, \xi_{2}\right\}$ determined by the last theorem the transversal frame associated to the null tangent frame $\left\{X_{1}, X_{2}\right\}$. Notice that if we interchange the fields $X_{1}$ and $X_{2}$, then the fields $\xi_{1}, \xi_{2}$ in the associated frame also interchange.

The following lemma gives the transformation rules for the associated frames.

Lemma 1.3. Let $\left\{X_{1}, X_{2}\right\}$ and $\left\{Y_{1}, Y_{2}\right\}$ be two null tangent frames. Let $\left\{\xi_{1}, \xi_{2}\right\}$ and $\left\{\eta_{1}, \eta_{2}\right\}$ be the respective associated transversal frames. Then there exists a $\mathbb{C}$-valued non-zero function $\gamma$ such that

$$
\begin{array}{ll}
Y_{1}=\gamma X_{1}, & Y_{2}=\gamma^{-1} X_{2}, \\
\eta_{1}=\gamma^{2} \xi_{1}, & \eta_{2}=\gamma^{-2} \xi_{2},
\end{array}
$$

after possibly interchanging $X_{1}$ and $X_{2}$, as well as $\xi_{1}$ and $\xi_{2}$, if necessary.

Assume that an affine metric $g$ is locally fixed. A complex-valued metric on an arbitrary transversal plane bundle $\sigma$ is defined in the following way. Let $u=\left\{X_{1}, X_{2}\right\}$ be a null frame and $\left\{\xi_{1}, \xi_{2}\right\}$ the associated transversal frame in $\sigma$. We define a metric $g_{u}^{\perp}$ on $\sigma$ by

$$
g_{u}^{\perp}\left(\xi_{1}, \xi_{1}\right)=g_{u}^{\perp}\left(\xi_{2}, \xi_{2}\right)=0, \quad g_{u}^{\perp}\left(\xi_{1}, \xi_{2}\right)=-2
$$

and extend it to a $\mathbb{C}$-bilinear symmetric form.

It turns out that $g_{u}^{\perp}$ is independent of the tangent frame $u$. Therefore we will denote it by $g^{\perp}$. Lemma 1.3 shows that for every $g^{\perp}$-null frame $\left\{\xi_{1}, \xi_{2}\right\}$ satisfying (1.17) there is a unique tangent $g$-null frame $\left\{X_{1}, X_{2}\right\}$ such that 
$\left\{\xi_{1}, \xi_{2}\right\}$ is associated to it. Therefore we will call both frames associated to each other.

The following theorem holds ([W], cf. [NV]):

TheOREM 1.5. For every complex, non-degenerate surface $M$ in $\mathbb{C}^{4}$ there is a unique equiaffine transversal bundle $\sigma$ such that $\nabla^{\perp} g^{\perp}$ is symmetric, where $g^{\perp}$ is an arbitrary transversal metric.

CoROllary 1.6. A transversal bundle $\sigma$ is the affine normal bundle if and only if the following equations hold:

$$
\begin{aligned}
& \nabla g\left(X_{1}, X_{2}, X_{1}\right)=\nabla g\left(X_{2}, X_{1}, X_{2}\right)=0, \\
& \nabla g\left(X_{1}, X_{1}, X_{1}\right)=\nabla g\left(X_{2}, X_{2}, X_{2}\right)=0,
\end{aligned}
$$

where $\nabla$ is the connection induced by $\sigma,\left\{X_{1}, X_{2}\right\}$ is a holomorphic null tangent frame and $g$ is the affine metric on the surface.

Corollary 1.7. Let $\sigma$ be a transversal bundle, $\left\{X_{1}, X_{2}\right\}$ a holomorphic null tangent frame, and $\left\{\xi_{1}, \xi_{2}\right\}$ the associated transversal frame. Then $\sigma$ is the affine normal bundle if and only if

$$
\tau_{1}^{1}+\tau_{2}^{2}=0, \quad \tau_{2}^{1}\left(X_{1}\right)=\tau_{1}^{2}\left(X_{2}\right)=0 .
$$

2. The proof of the main theorem. First we prove the following lemma:

Lemma 2.1. If the surface $M$ satisfies assumption 1) of Theorem 1.1, then for every point of $M$ there exists a neighbourhood and a null transversal frame $\left\{\xi_{1}, \xi_{2}\right\}$ such that the induced shape operators have the following properties:

$$
\nabla S_{1}=0, \quad \nabla S_{2}=0 .
$$

Proof. It suffices to define a new transversal frame $\left\{\widetilde{\xi}_{1}, \widetilde{\xi}_{2}\right\}$ by $(1.15)$ and (1.16) with $\gamma=e^{-\frac{1}{2} f}$.

In what follows we will use the transversal frame obtained in the previous lemma and the associated tangent frame $\left\{X_{1}, X_{2}\right\}$. Introduce local functions $a_{1}$ to $a_{8}$ by

$$
\begin{array}{ll}
\nabla_{X_{1}} X_{1}=a_{1} X_{1}+a_{2} X_{2}, & \nabla_{X_{1}} X_{2}=a_{3} X_{1}+a_{4} X_{2}, \\
\nabla_{X_{2}} X_{1}=a_{5} X_{1}+a_{6} X_{2}, & \nabla_{X_{2}} X_{2}=a_{7} X_{1}+a_{8} X_{2} .
\end{array}
$$

Using Corollary 1.6 we obtain

$$
a_{4}=-a_{1}, \quad a_{5}=-a_{8}, \quad a_{2}=0, \quad a_{7}=0 .
$$

The fact that $\nabla^{\perp} g^{\perp}=0$ implies that

$$
\tau_{2}^{1}=0 \quad \text { and } \quad \tau_{1}^{2}=0
$$


identically. If we define

$$
a=\tau_{1}^{1}\left(X_{1}\right), \quad b=\tau_{1}^{1}\left(X_{2}\right),
$$

we also have

$$
-a=\tau_{2}^{2}\left(X_{1}\right), \quad-b=\tau_{2}^{2}\left(X_{2}\right)
$$

using Corollary 1.7. The Codazzi equations (1.4) and (1.5) lead to

$$
a_{3}=-\left(b+2 a_{8}\right), \quad a_{6}=a-2 a_{1} .
$$

We introduce functions $c_{1}$ to $c_{4}$ as well as $k_{1}$ to $k_{4}$ such that

$$
\begin{array}{ll}
S_{1} X_{1}=c_{1} X_{1}+c_{2} X_{2}, & S_{1} X_{2}=c_{3} X_{1}+c_{4} X_{2}, \\
S_{2} X_{1}=k_{1} X_{1}+k_{2} X_{2}, & S_{2} X_{2}=k_{3} X_{1}+k_{4} X_{2} .
\end{array}
$$

In the next three lemmas we give some properties of these functions.

Lemma 2.2. The functions $c_{2}$ and $k_{3}$ vanish identically and $c_{3}=k_{2}$.

Proof. Since $\tau_{1}^{2}=0$, by (1.9) and (1.14) we have $0=-h^{2}\left(X_{2}, S_{1} X_{1}\right)=$ $-h^{2}\left(X_{2}, c_{1} X_{1}+c_{2} X_{2}\right)=-c_{2}$. Moreover (1.11), (1.14) and $\tau_{2}^{1}=0$ imply that $0=h^{1}\left(X_{1}, S_{2} X_{2}\right)=h^{1}\left(X_{1}, k_{3} X_{1}+k_{4} X_{2}\right)=k_{3}$. Using Corollary 1.7 and adding (1.8) and (1.10) we obtain $0=h^{1}\left(X_{1}, S_{1} X_{2}\right)-h^{2}\left(X_{2}, S_{2} X_{1}\right)=c_{3}-k_{2}$, which completes the proof.

Lemma 2.3. The following equations hold:

$$
b c_{1}=a c_{3}, \quad a c_{4}=0, \quad b k_{1}=0, \quad b k_{2}=a k_{4} .
$$

Proof. Since $\nabla S_{j}=0$, the Codazzi equations (1.6) and (1.7) imply

$$
\begin{aligned}
& 0=-b S_{1} X_{1}+a S_{1} X_{2}=\left(-b c_{1}+a c_{3}\right) X_{1}+\left(-b c_{2}+a c_{4}\right) X_{2}, \\
& 0=b S_{2} X_{1}-a S_{2} X_{2}=\left(b k_{1}-a k_{3}\right) X_{1}+\left(b k_{2}-a k_{4}\right) X_{2},
\end{aligned}
$$

and, by the previous lemma, we obtain (2.9).

Lemma 2.4. The coefficients $c_{j}$ and $k_{j}$, where $j=1,2$, satisfy

$$
\begin{aligned}
& X_{1}\left(c_{1}\right)=0 \\
& X_{1}\left(c_{3}\right)+2 a_{1} c_{3}-a_{3}\left(c_{1}-c_{4}\right)=0, \\
& X_{1}\left(c_{4}\right)=0 \\
& X_{2}\left(c_{1}\right)-a_{6} c_{3}=0 \\
& a_{6}\left(c_{1}-c_{4}\right)=0 \\
& X_{2}\left(c_{3}\right)-2 a_{8} c_{3}=0 \\
& X_{2}\left(c_{4}\right)+a_{6} c_{3}=0 \\
& X_{1}\left(k_{1}\right)+a_{3} k_{2}=0 \\
& X_{1}\left(k_{2}\right)-2 a_{1} k_{2}=0 \\
& -a_{3}\left(k_{4}-k_{1}\right)=0 \\
& X_{1}\left(k_{4}\right)-a_{3} k_{2}=0
\end{aligned}
$$




$$
\begin{aligned}
& X_{2}\left(k_{2}\right)+2 a_{8} k_{2}+a_{6}\left(k_{1}-k_{4}\right)=0, \\
& X_{2}\left(k_{1}\right)=0 \\
& X_{2}\left(k_{4}\right)=0 .
\end{aligned}
$$

Proof. The equations are consequences of the conditions $\nabla S_{j}=0$, applied to all possible pairs of the vector fields $X_{k}, X_{l}$, for $j, k, l=1,2$.

Substituting the triples $\left(X_{1}, X_{2}, X_{1}\right)$ and $\left(X_{1}, X_{2}, X_{2}\right)$ in the Gauss equation (1.3) and using Lemma 2.2 we obtain the following lemma.

Lemma 2.5. The functions $a_{1}, a_{3}, a_{6}$ and $a_{8}$ satisfy

$$
\begin{aligned}
& X_{1}\left(a_{8}\right)+X_{2}\left(a_{1}\right)=c_{3}-a_{6} a_{8}-a_{1} a_{3}-2 a_{1} a_{8}+a_{3} a_{6}, \\
& X_{1}\left(a_{6}\right)=-c_{4}+a_{6}\left(a_{1}-a_{6}\right), \\
& X_{2}\left(a_{3}\right)=-k_{1}+a_{3}\left(a_{8}-a_{3}\right) .
\end{aligned}
$$

We now define an open and dense subset of $M$ and we restrict our classification to this set. From the proof of Lemma 2.1 it follows that the frame defined there was defined up to constant non-zero factors. Thus the functions $a_{j}$ are also determined up to constant factors. Therefore the following definitions make sense on the whole surface, although they use local functions. Set

$$
U_{1}=\left\{p \in M \mid a_{3} \neq 0 \text { or } a_{6} \neq 0\right\}, \quad U_{2}=U \backslash \bar{U}_{1} .
$$

Then $U_{1} \cup U_{2}$ is an open and dense subset of $M$. We consider two cases.

Lemma 2.6. Any $p \in U_{1}$ has a neighbourhood $U_{p}$ in which $k_{2}=c_{3}=0$ identically, the functions $c_{1}, c_{4}, k_{1}$ and $k_{4}$ are constant, $c_{1}=c_{4}$ and $k_{1}=k_{4}$.

Proof. Let $U_{p}$ be contained in the domain of the frame with respect to which the functions $c_{j}$ and $k_{j}$ are defined. From the definitions of $U_{1}$ and the holomorphy of the functions considered it follows that $c_{1}=c_{4}$ or $k_{1}=k_{4}$ on the whole $U_{p}$.

We first assume that $c_{1}=c_{4}$. After adding (2.13) and (2.16) we have $X_{2}\left(c_{1}\right)=X_{2}\left(c_{4}\right)=0$ on $U_{p}$. Taking into account (2.10) and (2.12) we conclude that $c_{1}$ and $c_{4}$ are constant. Now (2.13) implies that $c_{3}=0$, and Lemma 2.2 gives $k_{2}=0$. If we consider the system (2.19), (2.21), then by the assumptions we have $k_{1}=k_{4}$. Using this fact and adding (2.17) and (2.20), we obtain $X_{1}\left(k_{1}\right)=X_{1}\left(k_{2}\right)=0$. Taking into account (2.22) and (2.23) now shows that $k_{1}$ and $k_{4}$ are constant. This completes the proof for $c_{1}=c_{4}$. The argument in the case when $k_{1}=k_{4}$ is analogous.

Lemma 2.7. There exists an open and dense subset $V$ of $U_{2}$ such that for every $p \in V$ there is a neighbourhood of $p$ that is affinely equivalent to 
one of the following surfaces:

$(2.28 \mathrm{a}) \quad x(u, v)=\left(u, v, u^{2}, v^{2}\right)$,

$(2.28 \mathrm{~b}) \quad x(u, v)=u \cdot\left(1, v, v^{2}, u^{2}\right)$,

$(2.28 \mathrm{c}) \quad x(u, v)=\left(u^{3}+3 u^{2} v, v^{3}+3 u v^{2}, u-v, u v\right)$,

$(2.28 \mathrm{~d}) \quad x(u, v)=(\sinh (u), \cosh (u), \sinh (v), \cosh (v))$,

(2.28e) $\quad x(u, v)=\left(v, \frac{1}{2} v^{2}, f_{1}(u), f_{1}(u) \int^{u} \frac{d s}{\left(f_{1}^{\prime}(s)\right)^{2}}-\int^{u} \frac{f_{1}(s) d s}{\left(f_{1}^{\prime}(s)\right)^{2}}\right)$,

where $f_{1}$ is an arbitrary holomorphic function of one variable defined in a neighbourhood of zero such that $f_{1}^{\prime} \neq 0$ and $f^{(3)}(0) \neq 0$.

Proof. By definition of $U_{2}, a_{3}=a_{6}=0$ identically. Using (2.10), (2.12), (2.13), (2.16), (2.17), (2.20), (2.22) and (2.23) we find that $c_{1}, c_{4}, k_{1}$ and $k_{4}$ are constant. By (2.25) and (2.26) we immediately obtain

$$
c_{4}=k_{1}=0 \text {. }
$$

We next use the Ricci equation (1.8) keeping the notation (2.5):

$$
c_{3}=X_{1}(b)-X_{2}(a)-\tau_{1}^{1}\left(\left[X_{1}, X_{2}\right]\right) \text {. }
$$

The Poisson bracket $\left[X_{1}, X_{2}\right]$ is equal to $\nabla_{X_{1}} X_{2}-\nabla_{X_{2}} X_{1}=-a_{1} X_{2}+a_{8} X_{1}$, and the last equation is equivalent to

$$
c_{3}=X_{1}(b)-X_{2}(a)+a_{1} b-a_{8} a \text {. }
$$

Considering the equation (2.7), we rewrite (2.29) as

$$
c_{3}=-2 X_{1}\left(a_{8}\right)-2 X_{2}\left(a_{1}\right)-4 a_{1} a_{8} .
$$

Next we consider the equation (2.24), which now has the form

$$
c_{3}=X_{1}\left(a_{8}\right)+X_{2}\left(a_{1}\right)+2 a_{1} a_{8} \text {. }
$$

Considering the system (2.30), (2.31), as well as Lemma 2.2, we obtain

$$
c_{3}=k_{2}=0 \text {. }
$$

We notice that the system (2.9) now has the form

$$
b c_{1}=0, \quad a k_{4}=0 .
$$

We define the following open subsets of $U_{2}$ :

$$
\begin{aligned}
& V_{1}=\left\{p \in U_{2} \mid c_{1} k_{4} \neq 0\right\}, \\
& V_{2}=\left(U_{2} \backslash \bar{V}_{1}\right) \cap\left\{c_{1} \neq 0 \text { or } k_{1} \neq 0\right\}, \\
& V_{3}=U_{2} \backslash\left(\bar{V}_{1} \cup \bar{V}_{2}\right) .
\end{aligned}
$$

Then $V_{1} \cup V_{2} \cup V_{3}$ is an open and dense subset of $U_{2}$. We notice that in $V_{3}$ both shape operators $S_{1}$ and $S_{2}$ are zero, so it is an umbilical surface considered in [VVW] in the real indefinite case. Since the argument and computations 
are very similar in our case, we conclude that there is an open and dense subset $W$ of $V_{3}$ which is affinely equivalent to one of the surfaces $(2.28 \mathrm{a}-\mathrm{c})$.

Now, $V_{1} \cup V_{2} \cup W$ is also an open and dense subset of $U_{2}$. Let $p \in V_{1}$. Then in a neighbourhood of $p$ we have $a=b=0$, by (2.33) and the definition of $V_{1}$. This implies

$$
a_{1}=a_{8}=0 \text {. }
$$

In this case we have $\nabla_{X_{j}} X_{k}=0$ for $j, k=1,2$, whence $\left[X_{1}, X_{2}\right]=0$. There are local complex coordinates $u$ and $v$ such that for the immersion $x$ the following equations hold:

$$
x_{u}=X_{1}, \quad x_{v}=X_{2} .
$$

By the Gauss formula (1.1) we obtain

$$
x_{u u}=\xi_{1}, \quad x_{u v}=0, \quad x_{v v}=\xi_{2} .
$$

This implies that $x_{u}$ depends only on $u$, and $x_{v}$ only on $v$. By the Weingarten formula we immediately obtain

$$
\begin{aligned}
& \left(\xi_{1}\right)_{u}=-c_{1} x_{u}, \\
& \left(\xi_{1}\right)_{v}=\left(\xi_{2}\right)_{u}=0, \\
& \left(\xi_{2}\right)_{v}=-k_{4} x_{v} .
\end{aligned}
$$

Combining (2.36) with (2.37) we obtain two differential equations:

$$
x_{u u u}=-c_{1} x_{u}, \quad x_{v v v}=-k_{4} x_{v} .
$$

Integrating them we have

$$
\begin{aligned}
& x_{u}=\cosh \left(\sqrt{-c_{1}} u\right) A+\sinh \left(\sqrt{-c_{1}} u\right) B, \\
& x_{v}=\cosh \left(\sqrt{-k_{1}} v\right) C+\sinh \left(\sqrt{-k_{1}} v\right) D,
\end{aligned}
$$

where $A, B, C$ and $D$ are constant vectors in $\mathbb{C}^{4}$. Up to an affine transformation we obtain the parametric equation

$$
x(u, v)=\left(\sinh \left(\sqrt{-c_{1}} u\right), \cosh \left(\sqrt{-c_{1}} u\right), \sinh \left(\sqrt{-k_{1}} v\right), \cosh \left(\sqrt{-k_{1}} v\right)\right) .
$$

If we treat $x$ as a function of the variables $\sqrt{-c_{1}} u$ and $\sqrt{-c_{1}} v$, we finally get the surface $(2.28 \mathrm{~d})$.

We consider the remaining case. Let $p \in V_{2}$. Let $c_{1} \neq 0$ first. When we change the null frame $\left\{X_{1}, X_{2}\right\}$ putting a constant function $\gamma$ such that $\gamma^{2}=c_{1}$ in (1.15), the functions $a$ and $b$ defined in (2.5) do not change. Thus we can assume that $c_{1}=1$. By definition of $V_{1}, k_{4}$ is equal to 0 . The first equation of (2.33) gives $b=0$, whence $a_{8}=0$. The equation (2.31) gives $X_{2}\left(a_{1}\right)=0$. Consider the system of differential equations

$$
X_{1}(\varrho)=a_{1} \varrho, \quad X_{2}(\varrho)=0
$$

for an unknown function $\varrho$ with $\varrho(p) \neq 0$. It is easy to see that the system satisfies the integrability conditions, so it has a solution. We define the vector 
fields $\widetilde{X}_{1}$ and $\widetilde{X}_{2}$ by the following formulas:

$$
\widetilde{X}_{1}=\frac{1}{\varrho} X_{1}, \quad \widetilde{X}_{2}=\varrho X_{2} .
$$

We then have $\nabla_{\widetilde{X}_{1}} \widetilde{X}_{2}=\varrho^{-1} X_{1}(\varrho) X_{2}+\nabla_{X_{1}} X_{2}=\varrho^{-1} a_{1} \varrho X_{2}-a_{1} X_{2}=0$ and, similarly, $\nabla_{\widetilde{X}_{2}} \widetilde{X}_{1}=0$. Thus there exist local coordinates $u$ and $v$ in a neighbourhood of zero such that $x(0,0)=p$ and

$$
x_{u}=\frac{1}{\varrho} X_{1}, \quad x_{v}=\varrho X_{2} .
$$

The frame $\left\{\widetilde{X}_{1}, \widetilde{X}_{2}\right\}$ is a null frame. The associated transversal frame $\left\{\widetilde{\xi}_{1}, \widetilde{\xi}_{2}\right\}$ is given by

$$
\widetilde{\xi}_{1}=\frac{1}{\varrho^{2}} \xi_{1}, \quad \widetilde{\xi}_{2}=\varrho^{2} \xi_{2} .
$$

To use the Gauss formula (1.1), we compute

$$
\nabla_{\widetilde{X}_{1}} \widetilde{X}_{1}=\frac{1}{\varrho} \nabla_{X_{1}}\left(\frac{1}{\varrho} X_{1}\right)=-\frac{1}{\varrho} \frac{X_{1}(\varrho)}{\varrho^{2}} X_{1}+\frac{1}{\varrho^{2}} a_{1} X_{1}=0 .
$$

Similarly, $\nabla_{\widetilde{X}_{1}} \widetilde{X}_{2}=0$ and $\nabla_{\widetilde{X}_{2}} \widetilde{X}_{2}=0$. Thus we have

$$
x_{u u}=\widetilde{\xi}_{1}, \quad x_{u v}=0, \quad x_{v v}=\widetilde{\xi}_{2} .
$$

The Weingarten formula implies

$$
\begin{aligned}
\left(\widetilde{\xi}_{1}\right)_{u} & =D_{\varrho^{-1} X_{1}}\left(\frac{1}{\varrho^{2}} \xi_{1}\right)=\frac{1}{\varrho} \frac{-2 X_{1}(\varrho)}{\varrho^{3}} \xi_{1}+\frac{1}{\varrho^{3}}\left(-S_{1} X_{1}+\tau_{1}^{1}\left(X_{1}\right) \xi_{1}\right) \\
& =\frac{-2 a_{1}}{\varrho^{3}} \xi_{1}-\frac{1}{\varrho^{3}} X_{1}+\frac{2 a_{1}}{\varrho^{3}} \xi_{1}=-\frac{1}{\varrho^{2}} \widetilde{X}_{1} \\
\left(\widetilde{\xi}_{1}\right)_{v} & =D_{\varrho X_{2}}\left(\frac{1}{\varrho^{2}} \xi_{1}\right)=\varrho \frac{-2 X_{2}(\varrho)}{\varrho^{3}} \xi_{1}+\frac{1}{\varrho}\left(-S_{1} X_{2}+\tau_{1}^{1}\left(X_{2}\right) \xi_{1}\right) \\
& =\frac{2 a_{8}}{\varrho} \xi_{1}=0 \\
\left(\widetilde{\xi}_{2}\right)_{u} & =D_{\varrho^{-1} X_{1}}\left(\varrho^{2} \xi_{2}\right)=\frac{1}{\varrho} 2 X_{1}(\varrho) \varrho \xi_{2}+\varrho\left(-S_{2} X_{1}+\tau_{2}^{2}\left(X_{1}\right) \xi_{2}\right) \\
& =2 a_{1} \varrho \xi_{2}-2 \varrho a_{1} \xi_{2}=0 \\
\left(\widetilde{\xi}_{2}\right)_{v} & =D_{\varrho X_{2}}\left(\varrho^{2} \xi_{2}\right)=\varrho \cdot 2 X_{2}(\varrho) \varrho \xi_{2}+\varrho\left(-S_{2} X_{2}+\tau_{2}^{2}\left(X_{2}\right) \xi_{2}\right)=0 .
\end{aligned}
$$

The equations (2.46), (2.47) and (2.43) imply that $x_{v v}$ is a constant vector. Hence

$$
x_{v}=v \cdot A+B
$$

for constant vectors $A, B \in \mathbb{C}^{4}$. By (2.42), (2.43), (2.45) and (2.46) we have

$$
x_{\text {uuu }}=-\varrho^{-2} x_{u} \text {. }
$$


We can treat (2.49) as a one-variable differential equation, homogeneous of order two. Its solution is of the form

$$
x_{u}=g_{1}(u) C+g_{2}(u) E,
$$

where $C$ and $E$ are elements of $\mathbb{C}^{4}$, and the functions $g_{1}$ and $g_{2}$ are linearly independent solutions of the differential equation

$$
y^{\prime \prime}+\varrho^{-2} y=0
$$

We can always choose $g_{1}$ and $g_{2}$ so that they are locally different from zero, which implies that the surface obtained by (2.48) and (2.49) is nondegenerate. (2.51) implies that their second derivatives are also different from zero. By the assumption, $a_{1}$ can be any function of $u$. Thus the function $\varrho(u)$ given by the equation $\varrho^{\prime}=a_{1}$ is arbitrary and non-zero in a neighbourhood of zero. Let $f_{1}$ and $f_{2}$ be indefinite integrals of $g_{1}$ and $g_{2}$, respectively. Since $x_{u}$ and $x_{u u}$ are linearly independent, the determinant $\left|\begin{array}{ll}f_{1}^{\prime} & f_{2}^{\prime} \\ f_{1}^{\prime \prime} & f_{2}^{\prime \prime}\end{array}\right|$ is different from zero.

On the other hand, $f_{1}^{\prime}$ and $f_{2}^{\prime}$ satisfy (2.51) and are non-zero, so

$$
f_{1}^{\prime \prime \prime} f_{2}^{\prime}-f_{1}^{\prime} f_{2}^{\prime \prime \prime}=0 \text {. }
$$

Using this equation, we compute

$$
\left(f_{1}^{\prime} f_{2}^{\prime \prime}-f_{2}^{\prime} f_{1}^{\prime \prime}\right)^{\prime}=f_{1}^{\prime \prime} f_{2}^{\prime \prime}+f_{1}^{\prime} f_{2}^{\prime \prime \prime}-f_{1}^{\prime \prime} f_{2}^{\prime \prime}-f_{1}^{\prime \prime \prime} f_{2}^{\prime}=0
$$

whence

$$
r:=f_{1}^{\prime} f_{2}^{\prime \prime}-f_{2}^{\prime} f_{1}^{\prime \prime}
$$

is a non-zero constant. We can see that each choice of $f_{1}$ with $f_{1}^{\prime}(0) \neq 0$ and $f_{1}^{(3)} \neq 0$ leads to an equation of a desired surface. Fix such an $f_{1}$. Then $(2.51)$ defines the function $\varrho$ explicitly. By (2.53), which we treat as a first order equation with respect to $f_{2}^{\prime}$, we obtain this function and its integral up to a constant. It has the form

$$
f_{2}(u)=r f_{1}(u) \int^{u} \frac{d s}{\left(f_{1}^{\prime}(s)\right)^{2}}-r \int^{u} \frac{f_{1}(s) d s}{\left(f_{1}^{\prime}(s)\right)^{2}} .
$$

Then by (2.48) and (2.50) and after applying an affine transformation we get the parametric equation (g) from the main theorem. The last case, $c_{1}=0$ and $k_{4} \neq 0$, can be reduced to the previous one by interchanging $X_{1}$ and $X_{2}$.

Notice that the surfaces we have obtained are essentially different, that is, not affinely equivalent. In the case of the umbilical surfaces this follows from the explanation given in [VVW]. For the other surfaces it follows from the fact that the shape operators are different and cannot coincide after admissible transformations of the null frames. 
Proof of Theorem 1.1. By (2.27) the set $U=U_{1} \cup U_{2}$ is open and dense in $M$. If $p \in U_{1}$, then by Lemma 2.6 it follows that there is a neighbourhood of $p$ which is an umbilical surface. Then from [VVW] (the real indefinite umbilical case) it follows that the neighbourhood is one of the surfaces (a) to (e). On the other hand, we can choose a transversal frame for which the shape operators satisfy $\nabla S_{1}=0, \nabla S_{2}=0$.

If $p \in U_{2}$, then Lemma 2.7 gives surfaces (a), (b), (c), (f) and (g). To prove the converse in this case it is enough to verify that the surfaces (f) and (g) satisfy assumption 1) of the theorem. For (f) we can see that the shape operators have constant coefficients with respect to the associated null tangent frame. This implies $\nabla S_{1}=\nabla S_{2}=0$. For (g) we choose a frame $\left\{\widetilde{X}_{1}, \widetilde{X}_{2}\right\}$ such that $\nabla_{\widetilde{X}_{j}} \widetilde{X}_{k}=0$ for all $j, k$. From $(2.44)-(2.47)$, we see that only $\widetilde{S}_{1} \widetilde{X}_{1}$ is non-zero and it is equal to $-\varrho^{-3} \widetilde{X}_{1}$. The equations $(2.1)$ are now satisfied with $f=-\varrho^{-3}$. This completes the proof of the theorem.

\section{References}

[A] K. Abe, Affine differential geometry of complex hypersurfaces, in: Geometry and Topology of Submanifolds III (Leeds, 1990), World Sci., Singapore, 1991, 1-31.

[DVV] F. Dillen, L. Verstraelen and L. Vrancken, Complex affine differential geometry, Atti Accad. Peloritana Pericolanti Cl. Sci. Fis. Mat. Nat. 66 (1988), 231-260.

[KN] S. Kobayashi and K. Nomizu, Foundations of Differential Geometry, Vols. I, II, Wiley, New York, 1963, 1969.

[NV] K. Nomizu and L. Vrancken, A new equiaffine theory for surfaces in $\mathbb{R}^{4}$, Internat. J. Math. 4 (1993), 127-165.

[OI] B. Opozda, Fundamental theorems for complex affine hypersurfaces, Kobe J. Math. 10 (1993), 133-146.

[OII] -, Equivalence theorems for complex affine hypersurfaces, Results Math. 27 (1995), 316-327.

[VVW] L. Verstraelen, L. Vrancken and P. Witowicz, Indefinite affine umbilical surfaces in $\mathbb{R}^{4}$, Geom. Dedicata 79 (2000), 109-119.

[W] P. Witowicz, Complex affine structures for surfaces in $\mathbb{C}^{4}$, Univ. Iagel. Acta Math. 40 (2002), 225-244.

Rzeszów Technical University

W. Pola 2

35-959 Rzeszów, Poland

E-mail: witowicz@prz.rzeszow.pl

Received 17.5.2006

and in final form 6.2.2007 